\title{
A HYPERCONTINUOUS HYPERSMOOTH SCHWARZSCHILD LINE ELEMENT TRANSFORMATION
}

\author{
ROBERT A. HERRMANN \\ Mathematics Department \\ United States Naval Academy \\ 572 Holloway Rd. \\ Annapolis, MD 21402-5002 USA
}

(Received April 3, 1995 and in revised form June 21, 1995)

\begin{abstract}
In this paper, a new derivation for one of the black hole line elements is given since the basic derivation for this line element is flawed mathematically. This derivation postulates a transformation procedure that utilizes a transformation function that is modeled by an ideal nonstandard physical world transformation process that yields a connection between an exterior Schwarzschild line element and distinctly different interior line element. The transformation is an ideal transformation in that in the natural world the transformation is conceived of as occurring at an unknown moment in the evolution of a gravitationally collapsing spherical body with radius greater than but near to the Schwarzschild radius. An ideal transformation models this transformation in a manner independent of the objects standard radius. It yields predicted behavior based upon a Newtonian gravitational field prior to the transformation, predicted behavior after the transformation for a field internal to the Schwarzschild surface and predicted behavior with respect to field alteration processes during the transformation.
\end{abstract}

KEY WORDS AND PHRASES. Eddington-Finkelstein transformation, hypercontinuous, hypersmooth, black hole metric, nonstandard analysis, nonstandard substratum.

1992 AMS SUBJECT CLASSIFICATION CODES. 83C57, 03H10.

\section{INTRODUCTION.}

In [1], the linear effect line element is derived and, in [2], a general line element $d S^{2}$ is derived by considering a non-reversible $P$-process emanating from the center of a spherical configuration and its interaction with the substratum. This interaction is modeled by taking the Special Theory chronotopic interval and modifying its spherical coordinate transformation by a type of damping of the basic light-clock counts. This damping is characterized by the two expressions (i) $d R^{m}=$ $d R^{s}+\alpha d T^{s}$ and (ii) $d T^{s}=\beta d R^{m}+d T^{m}$, where the $\alpha$ and $\beta$ are to be determined. From these determinations, the following general line element is derived.

$$
d S^{2}=\lambda\left(c d t^{m}\right)^{2}-(1 / \lambda)\left(d R^{m}\right)^{2}-\left(R^{s}\right)^{2}\left(\sin ^{2} \theta^{s}\left(d \phi^{s}\right)^{2}+\left(d \theta^{s}\right)^{2}\right) .
$$

The Eddington-Finkelstein transformation is the least ad hoc and is more physically justified than others. But, in [5], the derivation and argument for using the this simple transformation (1) 
$d U^{m}=d t^{m}+f_{M}\left(R^{m}\right) d R^{m}$ to obtain a black hole line element is flawed. This flaw is caused by the usual ad hoc logical errors in "removing infinities." Equation 57.11 in [5, p. 157], specifically requires that $R^{m}>2 G M / c^{2}$. However, in arguing for the use of the transformed Schwarzschild line element (1.1), Lawden assumes that it is possible for $R^{m}=2 G M / c^{2}$. But the assumed real valued function defined by equation 57.11 is not defined for $R^{m}$ such that $R^{m}=2 G M / c^{2}$. Since the derivation of the Schwarzschild line element in [2] does not require the General Principle of Relativity and, indeed, assumes that there is a privileged observer within a substratum, a new and rigorously correct procedure is necessary. This is accomplished by showing that (1) can be considered as a hypercontinuous and hypersmooth transformation associated with a new non-reversible $P$-process that yields an alteration to the gravitational field in the vicinity of the Schwarzschild surface during the process of gravitational collapse. This speculation is modeled by the expression (1) which is conceived of as an alteration in the time measuring light-clock. Further, this alteration is conceptually the same as the ultrasmooth microeffects model for fractual behavior [4]. This transformation takes the Schwarzschild line element, which applies only to the case where $R^{m}>2 G M / c^{2}$, and yields an NSP-world black hole line element that only applies for the case where $R^{m} \leq 2 G M / c^{2}$. Like ultrasmooth microeffects, the nonstandard transformation process is considered as an ideal model of behavior that approximates the actual natural world process. Thus we have two district line elements connected by such a transformation and each applies to a specific $R^{m}$ domain.

2. THE FUNCTION $f_{M}\left(R^{m}\right)$.

To establish that an internal function $f_{M}\left(R^{m}\right)$ exists with the appropriate properties proceed as follows: let $\mathcal{I}$ be the set of all nonsingleton intervals in $\mathcal{P}(\mathbb{R})$. Let $\mathcal{F} \subset \mathcal{P}(\mathbb{R} \times \mathbb{R})$ be the set of all nonempty functional sets of ordered pairs. For each $I \in \mathcal{I}$, let $C(I, \mathbb{R}) \subset \mathcal{F}$ be the set of all real valued continuous functions (end points included as necessary) defined on $I$. For each $a>0$, $\exists f_{a} \in C((-\infty, 0], \mathbb{R}),(-\infty, 0] \in \mathcal{I}$, such that $\forall x \in(-\infty, 0], f_{a}(x)=1 /(x-a)$. Further, $\exists g_{a} \in$ $C((0,2 a], \mathbb{R}),(0,2 a] \in \mathcal{I}$, such that $\forall x \in(0,2 a], g_{a}(x)=-x^{3} /\left(2 a^{4}\right)+7 x^{2} /\left(4 a^{3}\right)-x / a^{2}-1 / a$. Then $\exists h_{a} \in C((2 a,+\infty), \mathbb{R}),(2 a,+\infty) \in \mathcal{I}$, such that $\forall x \in(2 a,+\infty), h_{a}(x)=0$. Finally, it follows that $\lim _{x \rightarrow 0}-f_{a}(x)=\lim _{x \rightarrow 0+} g_{a}(x), \lim _{x \rightarrow 2 a}-g_{a}(x)=\lim _{x \rightarrow 2 a}+h_{a}(x)$. Hence

$$
H_{a}(x)= \begin{cases}f_{a}(x) ; & x \in(-\infty, 0] \\ g_{a}(x) ; & x \in(0,2 a] \\ h_{a}(x) ; & x \in(2 a,+\infty)\end{cases}
$$

is continuous for each $x \in \mathbb{R}$ and has the indicated properties.

Now $H_{a}^{\prime}(x)$ exists and is continuous for all $x \in \mathbb{R}$ and

$$
H_{a}^{\prime}(x)= \begin{cases}f_{a}^{\prime}(x) ; & x \in(-\infty, 0] \\ g_{a}^{\prime}(x) ; & x \in(0,2 a] \\ h_{a}^{\prime}(x) ; & x \in(2 a,+\infty)\end{cases}
$$

All of the above can be easily expressed in a first-order language and all the statements hold in our superstructure enlargement [4]. Let $0<\epsilon \in \mu(0)$. Then there exists an internal hypercontinuous hypersmooth $H_{\epsilon}:{ }^{*} \mathbb{R} \rightarrow{ }^{*} \mathbb{R}$ such that $\forall x \in{ }^{*}(-\infty, 0], H_{\epsilon}(x)=1 /(x-\epsilon)$ and $\forall x \in{ }^{*}(-\infty, 0) \cap \mathbb{R}$, st $\left(H_{\epsilon}(x)\right)=\operatorname{st}(1 /(x-\epsilon))=1 / x ;$ and for $x=0, H_{\epsilon}(0)$ exists, although $\operatorname{st}\left(H_{\epsilon}(0)\right)$ does not exist as a real number. Further, $\forall x \in(2 \epsilon,+\infty) \cap \mathbb{R}=(0,+\infty)$, st $\left(H_{\epsilon}(x)\right)=0$. To obtain the hypercontinuous hypersmooth $f_{M}$, simply let $c f_{M}=H_{\epsilon}, x=\lambda, R^{m} \in{ }^{*} \mathbb{R}$. 


\section{MOTIVATION FOR FUNCTION SELECTION.}

Recall that a function $f$ defined on interval $I$ is standardizable (to $F$ ) on $I$ if $\forall x \in I \cap$ $\mathbb{R}, F(x)=\operatorname{st}(f(x)) \in \mathbb{R}$. Now, consider the transformation (1) in the nonstandard form $d U^{m}=$ $d t^{m}+f_{M}\left(R^{m}\right) d R^{m}$ where internal $f_{M}\left(R^{m}\right)$ is a function defined on $A \subset{ }^{*} \mathbb{R}$, and $\lambda=\lambda\left(R^{m}\right)$. There are infinitely many nonstandard functions that can be standardized to produce the line element $d S^{2}$. In this line element, consider substituting for the function $\lambda=\lambda\left(R^{m},\right)$ the function ${ }^{*} \lambda-\epsilon$. The transformed line element then becomes, prior to standardizing the coefficient functions (i.e. restricting them the the natural world),

$$
\begin{gathered}
T=\left({ }^{*} \lambda-\epsilon\right) c^{2}\left(\left(d U^{m}\right)^{2}-2 f_{M} d U^{m} d R^{m}+f_{M}^{2}\left(d R^{m}\right)^{2}\right)-\left(1 /\left({ }^{*} \lambda-\epsilon\right)\right)\left(d R^{m}\right)^{2}- \\
\left(R^{m}\right)^{2}\left(\sin ^{2} \theta^{m}\left(d \phi^{m}\right)^{2}+\left(d \theta^{m}\right)^{2}\right)= \\
\left({ }^{*} \lambda-\epsilon\right) c^{2}\left(d U^{m}\right)^{2}-2\left({ }^{*} \lambda-\epsilon\right) c^{2} f_{M} d U^{m} d R^{m}+ \\
\overbrace{\left(\left({ }^{*} \lambda-\epsilon\right) c^{2} f_{M}^{2}-\left(1 /\left({ }^{*} \lambda-\epsilon\right)\right)\right) d R^{m}}^{{ }^{*} \lambda} d R^{m}- \\
\left(R^{m}\right)^{2}\left(\sin ^{2} \theta^{m}\left(d \phi^{m}\right)^{2}+\left(d \theta^{m}\right)^{2}\right) .
\end{gathered}
$$

Following the procedure outlined in [4], first consider the partition $\mathbb{R}=(-\infty, 0] \cup(0,2 \epsilon] \cup$ $(2 \epsilon,+\infty)$, where $\epsilon$ is a positive infinitesimal. Consider the required constraints. (2) As required, for specific real intervals, all coefficients of the terms of the transformed line element are to be standardized and, hence, are standard functions. (3) Since any line element transformation, prior to standardization, should retain its infinitesimal character with respect to an appropriate interval $I$, then for any infinitesimal $d R^{m}$ and for each value $R^{m} \in I$ terms such as $G\left(R^{m}\right) d R^{m}$, where $G\left(R^{m}\right)$ is a coefficient function, must be of infinitesimal value.

For the important constraint (3), Definition 4.1.1, and theorems 4.1.1, 4.1.2 in [3] imply that for a fixed infinitesimal $d R^{m}$ in order to have expression $b$ infinitesimal as $R^{m}$ varies, the coefficient $h\left(R^{m}\right)=\left({ }^{*} \lambda-\epsilon\right) c^{2} f_{M}^{2}-1 /\left({ }^{*} \lambda-\epsilon\right)$ must be infinitesimal on a subset $A$ of an appropriate interval $I$ such that $0 \in A$. The simplest case would be to assume that $A={ }^{*}(-\infty, 0]$. Let standard $r \in A \cap \mathbb{R}$. Then it follows that $h(r) \subset \mu(0)$. Thus $\operatorname{st}(h(r))=0$. Indeed, let $x \in(\cup\{\mu(r) \mid r<$ $0, r \in \mathbb{R}\}) \cup(\mu(0) \cap A)$. Then $\operatorname{st}(h(x))=0$. Since we are seeking a transformation process that is hypercontinuous, at least on ${ }^{*}(-\infty, 0]$, this last statement suggests the simplest to consider would be that on ${ }^{*}(-\infty, 0], h=0$. Thus the basic constraint yields the basic requirement that on ${ }^{*}(-\infty, 0]$ the simplest function to choose is $c f_{M}(x)=1 /(x-\epsilon)$. Since standardizing is required on ${ }^{*}(-\infty, 0) \cap \mathbb{R}$, we have for each $x \in \epsilon^{*}(-\infty, 0) \cap \mathbb{R}$, that $\operatorname{st}\left(c f_{M}(x)\right)=$ $\operatorname{cst}\left(f_{M}(x)\right)=\operatorname{st}(1 /(x-\epsilon))=1 / x$. This leads to the assumption that on $(-\infty, 0]$ the function $f_{a}(x)=1 /(x-a), a>0$, should be considered. After *-transferring and prior to standardizing, this selection would satisfy (3) for both of the coefficients in which $f_{M}$ appears and for the interval $I={ }^{*}(-\infty, 0]$. The function $g_{a}$ is arbitrarily selected to satisfy the hypercontinuous and hypersmooth property and, obviously, $h_{a}$ is selected to preserve the original line element for the interval $(2 \epsilon,+\infty)$. Finally, it is necessary that the resulting new coefficient functions, prior to standarizing, all satisfy (3) at least for a fixed $d R^{m}$ and a varying $R^{m} \in{ }^{*}(-\infty, 0]$ for the expression (1). It is not difficult to show that $\left|H_{\epsilon}(x)\right| \leq 2 / \epsilon$ for all $x \in{ }^{*} \mathbb{R}$. Consequently, for $\epsilon=\left(d R^{m}\right)^{1 / 3}$ expression (1) is an infinitesimal for all $R^{m} \in{ }^{*} \mathbb{R}$. 
Let $1-v^{2} / c^{2}=\lambda$. For the collapse scenario $R^{m}=R_{M}$. If $2 G M /\left(R^{m} c^{2}\right)<1$, substituting $2 G M / R^{m}=v^{2}$, into (1.1) yields the so-called Schwarzschild line element. With respect to the transformation, (A) if $R^{m}<2 G M / c^{2}$, then for st $\left(f_{M}\left(R^{m}\right)\right)=1 /(c \lambda), \lambda=1-2 G M /\left(R^{m} c^{2}\right)$; for (B) $R^{m}>2 G M / c^{2} ; \operatorname{st}\left(f_{M}\left(R^{m}\right)\right)=0$, and for the case that (C) $R^{m}=2 G M / c^{2}$, the function $f_{M}$ is defined and equal to a NSP-world value $f_{M}\left(R^{m}\right)$. But, for case (C), st $\left(f_{M}\left(R^{m}\right)\right)$ does not exist as a real number. Hence, $(C)$ has no direct effect within the natural world when $R^{m}=2 G M / c^{2}$, although the fact that $f_{M}\left(R^{m}\right) d R^{m}$ is an infinitesimal implies that $\operatorname{st}\left(f_{M}\left(R^{m}\right) d R^{m}\right)=0$. Using these NSP-world functions and (3.1), cases (A) and (C) yield

$$
\begin{aligned}
& d S_{1}^{2}=\lambda\left(c d U^{m}\right)^{2}-2 c d U^{m} d R^{m}- \\
& \left(R^{m}\right)^{2}\left(\sin ^{2} \theta^{m}\left(d \phi^{m}\right)^{2}+\left(d \theta^{m}\right)^{2}\right) .
\end{aligned}
$$

But case (B), leads to (1.1). The two constraints are met by $f_{M}\left(R^{m}\right)$, and indeed the standardized (A) form for $f_{M}\left(R^{m}\right)$ is unique if (3) is to be satisfied for a specific interval.

Since this is an ideal approximating model, in order to apply this ideal model to the natural world, one most select an appropriate real $a$ for the real valued function $H_{a}$. Finally, it is not assumed that the function $g_{a}$ is unique. In any solutions for line element (3.2), the $d U^{m}$ [resp. $d R^{m}$ ] refers to the timing [resp. length] infinitesimal light-clock counts and does refer to universal time [resp . length] alterations.

\section{REFERENCES}

1. HERRMANN, R.A. An operator equation and relativistic alterations in the time for radioactive decay, Intern. J. Math. \& Math. Sci., (to appear).

2. HERRMANN, R.A. Constructing Logically Consistent Special and General Theories of Relativity, Math. Dept., U.S. Naval Academy, Annapolis, MD, 1993.

3. HERRMANN, R.A. Some application of nonstandard analysis to undergraduate mathematics: infinitesimal modeling and elementary physics, Instructional Development Project, Mathematics Department, U. S. Naval Academy, Annapolis, MD, 21402-5002, 1991.

4. HERRMANN, R.A. Fractals and ultrasmooth microeffects, J. Math. Phys., 30(April 1989), 805-808.

5. LAWDEN, D. F. An Introduction to Tensor Calculus, Relativity and Cosmology, John Wiley \& Sons, New York, 1982. 


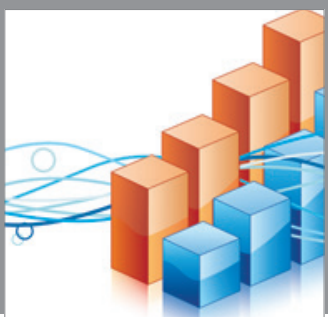

Advances in

Operations Research

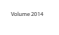

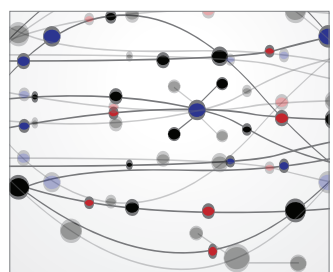

\section{The Scientific} World Journal
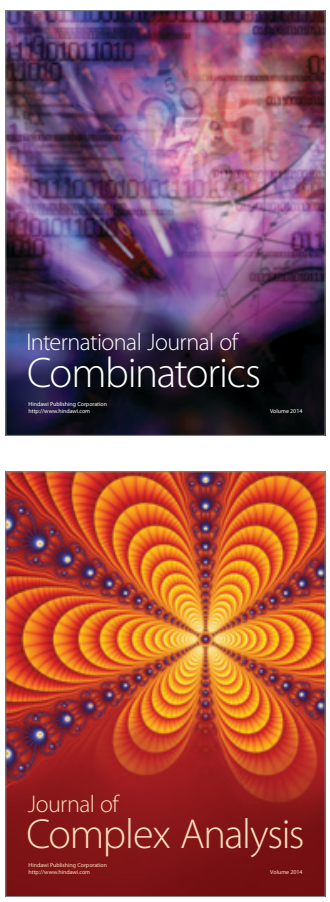

International Journal of

Mathematics and

Mathematical

Sciences
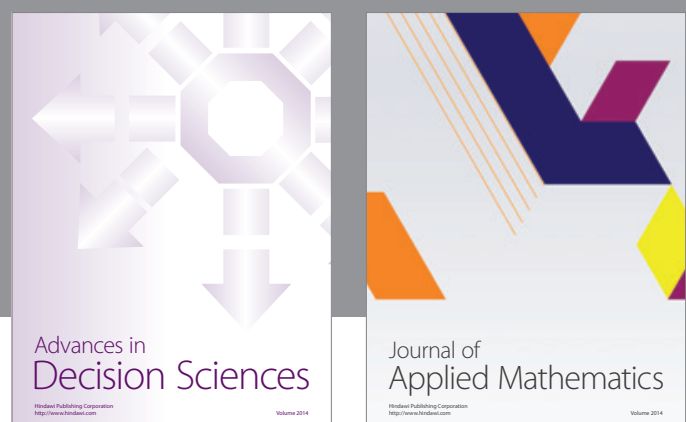

Journal of

Applied Mathematics
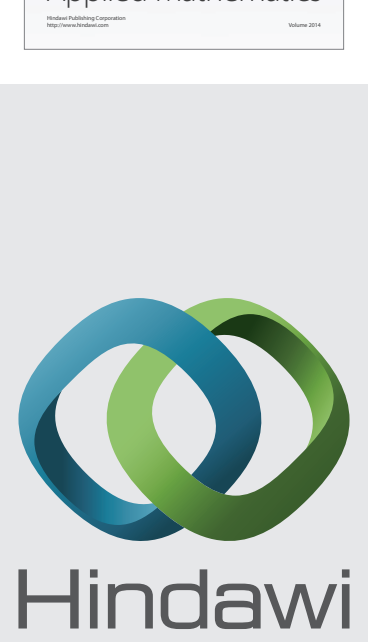

Submit your manuscripts at http://www.hindawi.com
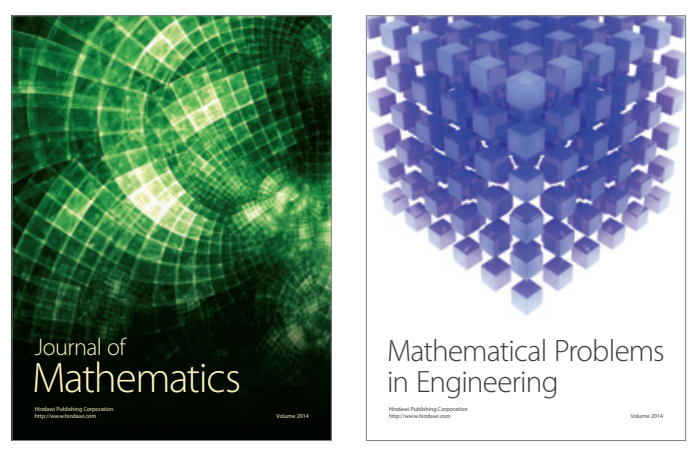

Mathematical Problems in Engineering
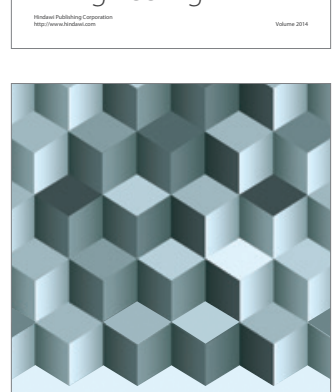

Journal of

Function Spaces
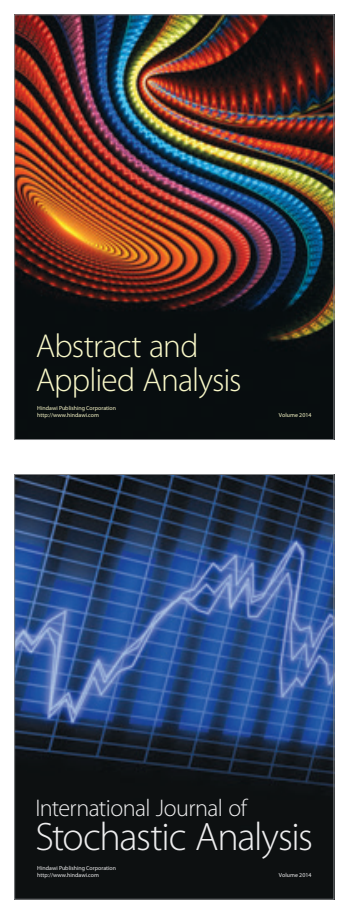

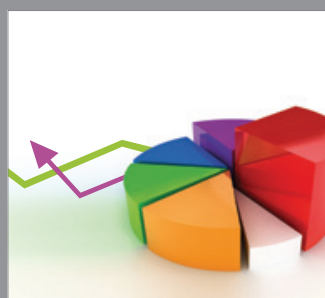

ournal of

Probability and Statistics

Promensencen
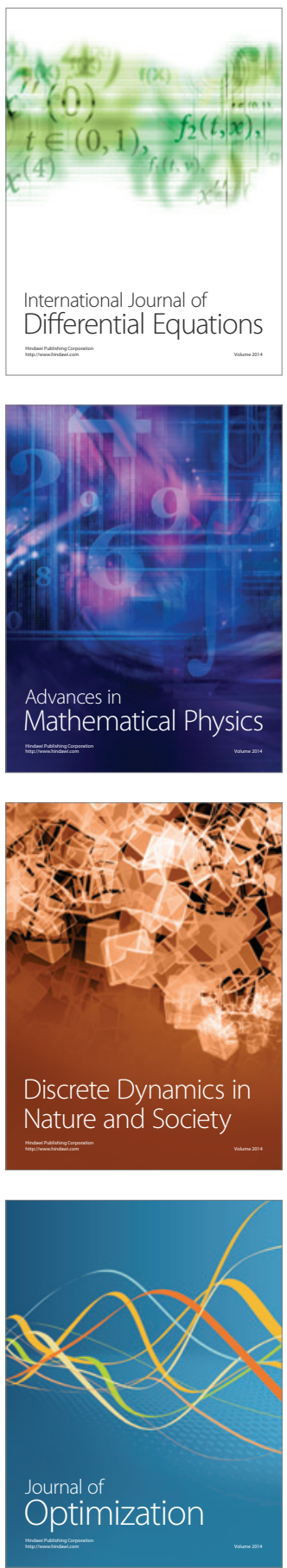